\title{
A influência digital no meio universitário - Sul de Minas Gerais
}

\author{
The digital influence in the university environment - South of Minas Gerais \\ La influencia digital en el ámbito universitario - Sur de Minas Gerais
}

Recebido: 22/07/2021 | Revisado: 27/07/2021 | Aceito: 07/08/2021 | Publicado: 11/08/2021

\author{
Bárbara Betina Lamana \\ ORCID: https://orcid.org/0000-0003-2112-0370 \\ Universidade José do Rosário Vellano, Brasil \\ E-mail: bblamana@gmail.com \\ Diego Vilela Amaral \\ ORCID: https://orcid.org/0000-0003-2531-8376 \\ Universidade José do Rosário Vellano, Brasil \\ E-mail: diegovilelaamaral@gmail.com \\ Gabryela Silveira de Lima Eleutério \\ ORCID: https://orcid.org/0000-0003-0144-7464 \\ Universidade José do Rosário Vellano, Brasil \\ E-mail: gabryelasle@gmail.com \\ Jordana Fernandes Almeida \\ ORCID: https://orcid.org/0000-0001-8344-5889 \\ Universidade José do Rosário Vellano, Brasil \\ E-mail: jordana.almeida@aluno.unifenas.br \\ Thaís Cardoso Machado \\ ORCID: https://orcid.org/0000-0001-5065-7236 \\ Universidade José do Rosário Vellano, Brasil \\ E-mail: thaismachado00@gmail.com \\ Ciderleia Castro de Lima \\ ORCID: https://orcid.org/0000-0002-7737-3935 \\ Universidade José do Rosário Vellano, Brasil \\ E-mail: ciderleiacastro@yahoo.com.br
}

\begin{abstract}
Resumo
Com a popularização da internet, o comportamento universitário tem sido modificado pelas Tecnologias de Informação e Comunicação (TICs). As redes sociais têm servido como meio de comunicação, além de contribuírem para o compartilhamento de conhecimento. Objetiva-se identificar como a tecnologia atua na vivência estudantil dos universitários, interferindo tanto nos estudos, quanto nas relações interpessoais. Para isso, aplicou-se um formulário online pela plataforma Google Forms, respondido por 148 estudantes de uma Universidade de ensino privado, no sul de Minas Gerais, de modo a compreender o perfil sociodemográfico e o uso das redes sociais pela amostra. Encontrou-se que as tecnologias digitais já estão bem difundidas no meio acadêmico, principalmente em tempos de isolamento social decorrente da pandemia do COVID-19, contribuindo tanto para a obtenção de conhecimento e entretenimento, quanto para a comunicação. A influência digital foi considerada benéfica no meio universitário, visto que contribuiu positivamente para os estudos, além de contribuir para o compartilhamento de informações e relações interpessoais à distância.
\end{abstract}

Palavras-chave: Aprendizado online; Tecnologias da informação e comunicações; Uso de redes sociais.

\begin{abstract}
With the popularization of the internet, university behavior has been modified by Information and Communication Technologies (ICTs). Social networks have served as a means of communication, besides contributing to the sharing of knowledge. The objective is to identify how technology acts in the student experience of university students, interfering both in their studies and in their interpersonal relationships. For this, an online form was applied through the Google Forms platform, answered by 148 students from a private university in southern Minas Gerais, in order to understand the sociodemographic profile and the use of social networks by the sample. It was found that digital technologies are already well disseminated in the academic environment, especially in times of social isolation resulting from the COVID-19 pandemic, contributing both for obtaining knowledge and entertainment, and for communication. Digital influence was considered beneficial in the university environment, as it contributed positively to studies, as well as contributing to information sharing and interpersonal relationships at a distance.
\end{abstract}

Keywords: Information and communications technologies; Online learning; Social networking. 


\begin{abstract}
Resumen
Con la popularización de Internet, el comportamiento universitario se ha visto modificado por las Tecnologías de la Información y la Comunicación (TIC). Las redes sociales han servido como medio de comunicación, además de contribuir a compartir conocimientos. El objetivo es identificar cómo actúa la tecnología en la experiencia estudiantil de los universitarios, interfiriendo tanto en los estudios como en las relaciones interpersonales. Para ello, se aplicó un formulario online mediante la plataforma Google Forms, respondieron 148 estudiantes de una universidad privada del sur de Minas Gerais, con el fin de conocer el perfil sociodemográfico y el uso de las redes sociales por parte de la muestra. Se comprobó que las tecnologías digitales ya están bien difundidas en el ámbito académico, especialmente en tiempos de aislamiento social derivados de la pandemia de COVID-19, contribuyendo tanto a la obtención de conocimientos y entretenimiento, como a la comunicación. La influencia digital se consideró beneficiosa en el entorno universitario, ya que contribuyó positivamente a los estudios, además de contribuir a compartir información y relaciones interpersonales a distancia.
\end{abstract}

Palabras clave: Aprendizaje en línea; Red social; Tecnologías de la información y la comunicación.

\title{
1. Introdução
}

A Terceira Revolução Industrial, decorrente da $2^{a}$ Guerra Mundial, também conhecida como Revolução TécnicoCientífica-Informacional, propiciou o surgimento de novas tecnologias, como a informática, a robótica, avanços nas áreas de telecomunicação e de eletrônica, entre outras. Após a revolução industrial o corpo humano se expandiu por causa do telescópio, do telégrafo, da máquina a vapor, do telefone, do rádio, da televisão etc. Agora com os satélites e a informática, o nosso cérebro se expande diminuindo distâncias espaciais e intervalos temporais até abolir o espaço e o tempo. De fato, o universo está online durante vinte e quatro horas, sem obstáculos de distância e de diferenças geográficas, diferenças sociais, diferenças políticas, nem com a distinção entre o dia e a noite, ontem e amanhã. Tudo se passa aqui e agora (Chauí, 2010).

Nesse contexto, observa-se a expansão da tecnologia pela sociedade moderna, ganhando cada vez mais espaço no cotidiano dos indivíduos. Dessa forma, não só as relações interpessoais estão mudando e se adaptando, mas também o jeito com que o indivíduo observa e analisa o mundo. A grande plataforma online facilita a obtenção de conhecimento, visto que porta imensa quantidade de informações que podem ser acessadas na comodidade do lar, a qualquer momento, com apenas alguns "cliques".

O mundo "virtual", tão presente nos relacionamentos sociais na sociedade atual, modificou qualitativa e quantitativamente, entre mais, a temporalidade, a articulação entre passado, presente e futuro. Como tudo acontece aqui e agora, há uma promessa de otimização do tempo e do espaço que se atualiza constantemente através de diversas mercadorias e recursos disponíveis no âmbito das tecnologias de comunicação e informação (Ramos, 2018).

A influência digital no comportamento universitário tem sido um assunto crescente na atualidade, especialmente pela popularização da internet. Com ela surgiram as redes sociais (virtuais), as quais criaram um cenário mais democrático para a difusão de informação, pois anteriormente este era obtido através de meios de informação físicos, como livros e jornais, ou até mesmo por meio de conhecimento científico repassado por profissionais da área da educação. Além disso, as ferramentas digitais, criadas e aprimoradas constantemente na atualidade, são outros meios que influenciam na difusão do conhecimento e nas facilidades existentes hoje para se aprimorar, complementar e completar uma formação (Souza \& Simon, 2018).

O filósofo alemão Jurgen Habermas defendeu em uma entrevista para a revista El País (2018) que a internet abriu milhões de nichos subculturais úteis, nos quais é possível trocar informação confiável e opiniões fundamentadas: “pensemos não só nos blogs dos cientistas, mas nos pacientes que sofrem de uma doença rara e entram em contato com outros na mesma condição em outro continente para se ajudar mutuamente com conselhos e experiências."

Portanto, a internet é uma ferramenta que contribui para a formação universitária, configurando-se um meio de pesquisa com alta renovação de conhecimento científico, que abrange praticamente todo o mundo, facilitando as trocas de informações, encurtando as distâncias e contribuindo para a formação de ideias e pensamentos dos indivíduos.

Nesse sentido, o objetivo deste estudo foi analisar como a tecnologia digital se faz presente na experiência de jovens 
universitários e assim compreender como esta interfere nas relações que esses jovens estabelecem entre si e com seus estudos. Buscou-se identificar o que ocorre nesta forma de experiência em que o acesso ao mundo virtual interfere no aprendizado e na obtenção de conhecimento da amostragem em estudo.

O uso das Tecnologias de Informação e Comunicação (TIC) vem provocando alterações nas relações entre as competências intelectuais e os conhecimentos, oportunizando mudanças de paradigmas em situações de aprendizagem. Isso afeta diretamente as relações de ensino e de aprendizagem, trazendo reflexos para as modalidades de educação, sendo necessário refletir o impacto dessa modernidade na sociedade e nos campos de ensino (Galafassi, 2013; Bierhalz et al., 2019).

\section{Metodologia}

Trata-se de um estudo epidemiológico transversal descritivo e de abordagem quantitativa, preconizado por Pereira et al. (2018). Assim, realizou-se uma pesquisa de campo com a finalidade de compreender o perfil dos universitários, na busca por identificar as influências de redes sociais na formação acadêmica.

A pesquisa foi realizada em uma Universidade de ensino privado, no sul de Minas Gerais, no primeiro semestre de 2021. Calculada uma amostragem de 380 universitários, desses, 148 estudantes de graduação participaram, de diferentes áreas e faixas etárias. Aplicou-se um formulário com perguntas semiestruturadas, elaborado pelos pesquisadores. $\mathrm{O}$ instrumento para coleta de dados contempla as variáveis de caracterização dos universitários desde informações sociodemográficas a acadêmicas.

Para abordagem aos participantes da pesquisa foi disponibilizado via online, por meio das redes sociais, o termo de consentimento livre e esclarecido, contendo as informações acerca dos objetivos da pesquisa e garantindo-lhes a confidencialidade de seus dados pessoais. O acesso ao formulário da pesquisa ficou condicionado ao aceite digital. É importante ressaltar que esse formulário foi disponibilizado pela plataforma do Google Forms e a identidade dos participantes mantida em sigilo conforme resolução 466/12 da CNS (Brasil, 2012).

Os dados obtidos foram organizados no software Microsoft Excel e, em seguida, realizou-se uma análise estatística descritiva. Os resultados estão apresentados em tabelas e gráficos. Com base nos resultados da análise, pretendeu-se elaborar um plano de orientação do tipo projeto de extensão para apoio psicológico integral.

A pesquisa foi iniciada após a apreciação do comitê de ética em pesquisa da Universidade, sob parecer n 4.402.206.

\section{Resultados}

Foram obtidas 148 respostas no questionário online, o qual ficou disponível entre os dias 1 a 16 de março de 2021. Todos os participantes aceitaram o Termo de Consentimento Livre e Esclarecido anexado no formulário, concordando em participar da pesquisa.

A grande maioria dos participantes tinha entre $18-24$ anos $(88,5 \%)$, se declararam brancos $(83,8 \%)$, solteiros $(95,9 \%)$, do sexo feminino (73,6\%), heterossexuais $(88,5 \%)$ e católicos $(63,5 \%)$. É importante ressaltar que cerca de $98 \%$ dos alunos estão cursando sua primeira graduação, sendo o curso de Medicina o mais presente na amostra (63,5\%), seguido pelos cursos de Enfermagem (15,5\%) e Odontologia (10,8\%). Todos os participantes afirmaram possuir conta em redes sociais, tais como Whatsapp (100\%), Instagram (98,6\%), Zoom (23\%), Facebook (83,1\%), Google Meet (92,6\%), Telegram (75,7\%), Twitter $(50,7 \%)$ e Snapchat $(34,5 \%)$. Outras plataformas digitais também foram citadas em menor frequência, como Linkedin, YouTube, Google Hangouts, Skype e Tik Tok, como demonstrado no Gráfico 1. 
Gráfico 1. Principais aplicativos utilizados pelos estudantes da amostra.

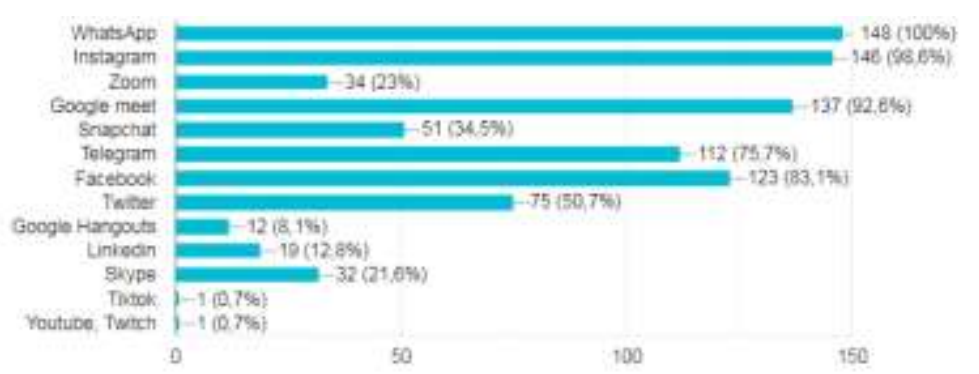

Fonte: Autores (2021).

Dos participantes, $95,9 \%$ da amostra relatou utilizar sempre as redes sociais, $98 \%$ com hábitos diários de uso. As redes sociais são utilizadas, principalmente, para distração e entretenimento (98\%). Em seguida, relatou-se o uso para bate papo $(87,2 \%)$, para estudos $(86,5 \%)$, para informação $(73,6 \%)$ e para trabalho $(36,5 \%)$, exibido no Gráfico 2.

Gráfico 2. Os principais motivos pelos quais as redes sociais são utilizadas pelos estudantes da amostra.

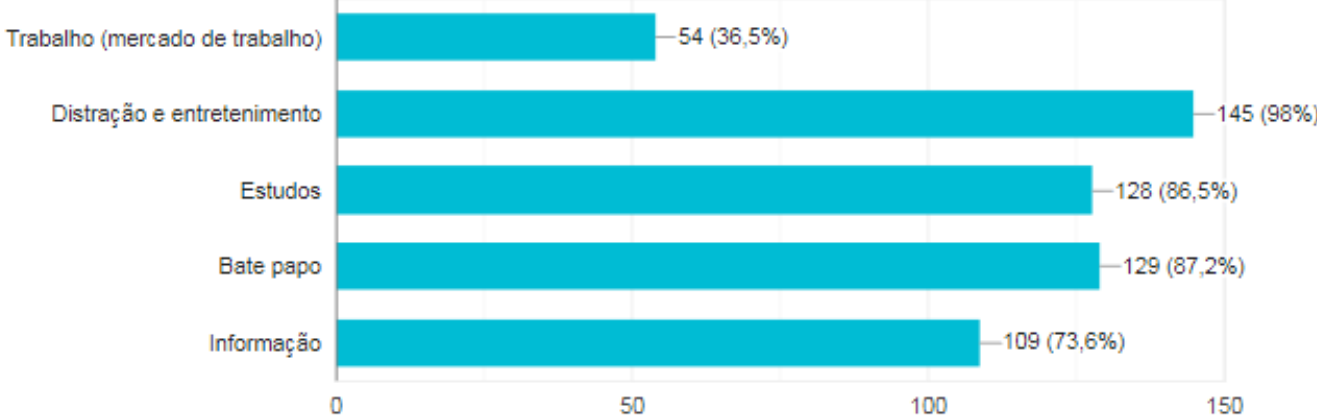

Fonte: Autores (2021).

Ao serem questionados sobre os hábitos de leitura em livros acadêmicos, 68,9\% dos participantes afirmaram que sempre buscam conhecimento em livros da área de sua formação. Quanto à frequência de idas à biblioteca da Universidade nos últimos 6 meses, 52,7\% dos alunos relataram que às vezes vão, enquanto 37,8\% relataram nunca terem ido. O fato pode ser explicado devido à possibilidade de acesso online à biblioteca da Universidade, pela plataforma Sophia. Além disso, o período entre 2020 e 2021 estava com restrições sociais devido à pandemia da COVID-19, contribuindo para que uma minoria dos alunos entrevistados frequentasse sempre a biblioteca $(9,5 \%)$. O rendimento acadêmico foi avaliado como bom, mas com maior dedicação, poderia ter sido melhor (40,5\%), como demonstrado no Gráfico 3.

Hábitos extracurriculares, como saídas em grupo para distração, foram frequentes em 43,2\% dos alunos. 31,1\% relataram que saem às vezes, $18,2 \%$ relataram que as saídas são apenas nos finais de semana, enquanto 7,4\% admitiram que nunca saem. Quando questionados sobre trabalho concomitante aos estudos, a grande maioria negou vínculo empregatício $(74,3 \%)$, podendo se dedicar apenas aos estudos. 
Gráfico 3. Avaliação dos alunos quanto ao rendimento acadêmico no último semestre.

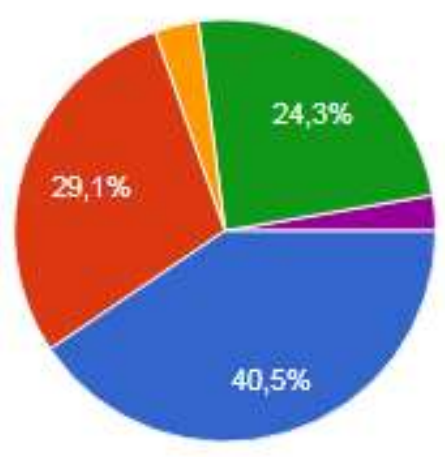

Bom, mas poderia ser melhor se dedicasse mais

Ótimo, mas ainda ficaram coisas pendentes

Excelente, absorveu o conhecido que foi passado

Regular, deixou-se levar por distraçôes e não estudou o suficiente

- Ruim, não conseguiu acompanhar a turma em várias disciplinas

Fonte: Autores (2021).

\section{Discussão}

O presente estudo tem como objetivo analisar o perfil sociodemográfico dos estudantes universitários de uma cidade localizada no sul de Minas Gerais, bem como o uso das ferramentas digitais. A população encontrada como amostra vai ao encontro com a pesquisa de Barros (2015), o qual descreveu a população universitária brasileira como, em sua maioria, branca, com idade entre 18 e 24 anos, predominando as universidades particulares. Já a predominância do sexo feminino da amostra condiz com registros do IBGE (Instituto Brasileiro de Geografia e Estatística), os quais relataram um aumento de 53,6\% para $57,1 \%$ da ocupação de vagas universitárias pelas mulheres, entre os anos de 2008 e 2018. Os nascidos após 1980 são conhecidos como "Nativos Digitais", devido ao convívio desde o nascimento com dispositivos tecnológicos (Prenski, 2001). Dessa forma, espera-se que essa geração tenha maior familiaridade e domínio sobre as Tecnologias de Informação e Comunicação (TIC's), as quais vêm sendo empregadas na educação (Chiusoli et al., 2020).

Todos os estudantes da amostra relataram possuir redes sociais, sendo o Whatsapp o mais usado. Tais dados corroboram com o estudo de Moromizato (2017), realizado no Sergipe, em que os estudantes analisados também relataram uso diário das plataformas digitais. Entre os motivos para essa popularização, destaca-se a idade dos alunos e a facilidade que estes têm em manusear aparelhos eletrônicos, buscando por informações necessárias, compartilhando conhecimento e se comunicando com pessoas próximas e de todo o mundo (Fermann et al., 2021).

O uso diário da internet também foi encontrado na grande maioria dos estudantes analisados por Rangel \& Miranda (2016), sendo que a metade deles afirmou ter um tempo de tela superior a 6 horas diárias. Em contrapartida, a minoria da amostra relatou um tempo de estudo superior a essas 6 horas gastas por dia na internet. Ao serem questionados sobre a intervenção das redes sociais nos estudos, 41,6\% acreditavam que estas não atrapalhavam o rendimento acadêmico, enquanto $27 \%$ acreditavam num impacto negativo e 31,4\% num impacto positivo. Concordando com esse cenário, dos estudantes analisados nesta pesquisa, 98\% afirmaram acessar todos os dias as redes sociais. Os estudantes mencionaram que, para que o rendimento acadêmico não fosse prejudicado, foi necessário conciliação entre atividades de lazer, sono e estudos (Rangel \& Miranda, 2016).

Distração e entretenimento foram as causas mais citadas pela amostra para o uso das redes sociais, seguidas dos estudos. Rangel \& Miranda (2016) descreveram que o lazer foi a principal causa para o uso das plataformas online, seguido pelas pesquisas. Por conta do tempo demandado para a dedicação aos estudos, é comum os universitários dedicarem pouco tempo a atividades de lazer. Esse cenário influencia diretamente na saúde mental e física dos estudantes, aumentando sintomas de estresse, ansiedade e depressão, os quais podem interferir no rendimento acadêmico (Fermann et al., 2020; Mota et al., 2021).

Além de serem utilizadas como passatempo, as redes sociais também são utilizadas para bate papos e comunicação. 
Como encontrado na presente amostra, $87,2 \%$ dos estudantes utilizam-nas para esta finalidade. As redes sociais estão amplamente presentes nas sociedades contemporâneas, permitindo uma globalização comunicacional (Angelo, 2016).

Apesar de 43,2\% da amostra ter afirmado sempre sair em grupo como modo de distração, no ano de 2020 as relações interpessoais físicas foram impossibilitadas pela pandemia do coronavírus e pelas medidas de contenção, sendo a principal delas, o isolamento social. Nesse contexto, as redes sociais tiveram grande importância na construção e manutenção de laços afetivos entre os jovens, incluindo os universitários (Xavier et al., 2020). Soares et al. (2016) afirmou que as relações sociais firmadas durante o período universitário são relevantes para a adaptação, para as vivências e para o rendimento acadêmico satisfatório.

Uma minoria dos universitários mineiros entrevistados alegou trabalho concomitante aos estudos (25,7\%), visto que para esse estudo o maior número de participantes foram acadêmicos de Medicina. O curso de Medicina exige de seus alunos uma dedicação em tempo integral, o que dificulta articular trabalho e estudos. Os dados encontrados discordaram do levantamento descrito por Silva \& Vizotto (2013), os quais afirmaram que, de acordo com o Instituto Data Popular de 2012, 7 entre cada 10 universitários estava vinculada a uma ocupação no mercado de trabalho, visto que para esse estudo englobou diversos perfis acadêmicos.

Dos 11 participantes que afirmaram nunca saírem em grupo para se distraírem, 5 afirmaram trabalhar juntamente com os estudos, todos esses pertencentes ao curso de Enfermagem. Ao mesmo tempo, as redes sociais pareceram ser uma alternativa para distração e interação para os universitários assalariados, visto que todos os 11 usam a internet para distração e entretenimento e/ou para bate papo.

Fermann et al. (2021) declara que a internet está cada vez mais presente na vida dos universitários brasileiros, podendo substituir livros físicos e bibliotecas quando o assunto é obtenção de conhecimento e compartilhamento de informação. Tal cenário é descrito pela amostra desta pesquisa, visto que a maioria dos estudantes relata não irem à biblioteca da Universidade com frequência, no entanto, a maioria da amostra afirmou buscar conhecimento em livros de sua área de formação. Os estudantes relatam que a plataforma digital auxilia no processo de aprendizagem, facilitando a pesquisa e o acesso a livros e a conteúdos de forma rápida e instantânea, possuindo potencialidades para enriquecer o processo de ensinoaprendizagem (Ramos, 2018; Nascimento \& Gomes, 2020).

Durante a pesquisa, o Brasil encontrava-se em período de isolamento social, com aulas e atividades presenciais interrompidas devido a pandemia do coronavírus. A Medida Provisória número 934, emitida pelo Ministério da Educação, permitiu a migração do ensino presencial para o ensino remoto, visando reduzir os impactos negativos da pandemia na educação (Brasil, 2020). Dessa forma, o ensino só foi possibilitado pelo uso de plataformas digitais e Tecnologias de Informação e Comunicação (TIC's). Dentre as plataformas mais utilizadas, descritas no estudo de Lamana et al (2020), encontram-se Google Meet, Google Forms, Moodle e Zoom. Tais ferramentas são capazes de atuar no compartilhamento de ideias e construção de novos aprendizados, proporcionando novos planejamentos por parte dos docentes (Carneiro et al., 2020).

Também foi descrito pela maioria dos estudantes analisados no estudo de Lamana et al (2020) que o rendimento não foi bom, contrapondo o encontrado na pesquisa atual, cuja maior parte dos estudantes analisados afirmam que os estudos no último semestre foram bons ou ótimos. Essa divergência demonstra como as consequências do uso de plataformas online é variável, devendo ser mais bem estudado qual o limite entre os benefícios e os malefícios do uso de ferramentas digitais nos estudos.

\section{Conclusão}

É sabido que as mídias sociais e os aparelhos digitais são parte do cotidiano da grande maioria da população brasileira 
e mundial. Estão presentes para fins de comunicação, de aprendizado, de compartilhamento de informações e de entretenimento. Mesmo que a maioria tenha alegado o uso de redes sociais para fins de bate papo, quase a mesma quantidade de alunos afirmou usar para estudos. Tal fato demonstra que a influência digital tem sido positiva no meio universitário, auxiliando nos estudos e na comunicação entre os estudantes.

Nesse sentido, sugere-se que futuros estudos investiguem até onde essa influência se mantém positiva nos estudos universitários, sem interferir de maneira negativa na saúde mental, nos estudos e nas relações interpessoais dos estudantes.

Como limitações, os pesquisadores encontraram dificuldade em obter respostas no questionário, sendo que estas ficaram concentradas na área de cursos da saúde. Dessa forma, não foi possível avaliar grandes diferenças quanto ao uso das redes sociais entre diversas áreas de formação superior.

\section{Referências}

Almeida, M. (2010). Tecnologia e Educação a distância: Abordagens e contribuições dos ambientes digitais e interativos de aprendizagem. Revista Brasileira de Educação a Distância.

Angelo, E. (2016). Redes sociais virtuais na sociedade da informação e do conhecimento: economia, poder e competência informacional. Revista eletrônica de biblioteconomia e ciência da informação, 21(46), 71-80.

Barros, A. S. X. (2015). Expansão da educação superior no brasil: limites e possibilidades. Educação \& Sociedade, 36(131), 361-390.

Bierhalz, C. D. et al. (2019). Concepções dos estudantes de uma escola do campo sobre tecnologia. Revista Brasileira de Educação do Campo, 4, 1-21.

Brasil, Ministério da Educação. Medida provisória n ${ }^{\circ}$ 934, de $1^{\circ}$ de abril de 2020. Diário Oficial da República Federativa do Brasil. Poder executivo, Brasília, DF.

Brasil, Ministério da Educação. Portarias n 343 e 345 de 2020.

Carneiro, L. de A. et al. (2020). Uso de tecnologias no ensino superior público brasileiro em tempos de pandemia COVID-19. Research, Society and Development, 9(8), e267985485.

Chauí, M. (2010). A contração do tempo e do espaço do espetáculo. Café Filosófico. http://www.youtube.com/watch?v=X5d1TBpXrq0ção

Chiusoli, C. L.et al. (2020). Atividade acadêmica, tecnologia e rede social: o comportamento da geração Z. Research, Society and Development, 9(3), e169932735.

Fermann I. L. et al. (2021). Uso de internet e mídias sociais por estudantes universitários: um campo de estudo emergencial. Ciências Psicológicas, 15(1), e2389.

Galafassi, F. et al. (2013). Análise crítica das pesquisas recentes sobre as tecnologias de objetos de aprendizagem e ambientes virtuais de aprendizagem. Revista Brasileira de Informática na Educação, 21(3), 41-52.

Habermas, J. (2018). Não pode haver intelectuais se não há leitores. El País. https://brasil.elpais.com/brasil/2018/04/25/eps/1524679056_056165.html

IBGE. Educação melhora, mas ainda apresenta desafios. Síntese dos Indicadores Sociais de 2008. http://saladeimprensa.ibge.gov.br/noticias?view =noticia\&i $\mathrm{d}=1$ \&busca=1\&idnoticia $=1233$.

Lamana, B. B. et al. (2020). Estudos universitários durante o isolamento social: pesquisa de opinião pública. Ebook I Congresso Nacional de Inovação e Popularização da Ciência, 1, 135-136.

Morozimato, M. S. et al. (2017). O Uso de Internet e Redes Sociais e a Relação com Indícios de Ansiedade e Depressão em Estudantes de Medicina. Revista Brasileira de Educação Médica, 41(4), 497- 504.

Mota, D. C. B. et al. (2021). Saúde mental e uso de internet por estudantes universitários: estratégias de enfrentamento no contexto da COVID-19. Ciência \& Saúde Coletiva, 26(6), $2159-2170$.

Nascimento, M. C. \& Gomes, G. R.R. (2020). Formação continuada docente para a utilização das TIC no processo de ensino e aprendizagem. Research, Society and Development, 9(2), e33921998.

Pereira, AS et al. (2018). Metodologia da pesquisa científica. UFSM. https://repositorio.ufsm.br/bitstream/handle/1/15824/Lic_Computacao_MetodologiaPesquisa-Cientifica.pdf?sequence $=1$.

Prensky, M. (2001). Digital natives, digital immigrants, part 1. On the horizon, 9(5), p. 1-6.

Ramos, V. A. (2018). Tecnologia e formação: o smartphone na experiência de jovens universitários - Universidade Federal de Goiás, Goiânia.

Rangel, J. R. \& Miranda, G. J. (2016). Desempenho acadêmico e o uso das redes sociais. Sociedade, Contabilidade e Gestão, 11(2), 139-154.

Silva, F. C. \& Vizzotto, M. M. (2013). Perfil do estudante universitário usuário de tecnologias. Psicólogo Informação, 7(17), 39-55. 
Research, Society and Development, v. 10, n. 10, e300101018598. 2021

(CC BY 4.0) | ISSN 2525-3409 | DOI: http://dx.doi.org/10.33448/rsd-v10i10.18598

Soares, A. B. et al. (2016) Relações interpessoais na universidade: o que pensam estudantes da graduação em psicologia? Estudos Interdisciplinares em Psicologia, 7(1), 56-76.

Souza, M. V. \& Simon, R. M. (2018). Redes sociais e moocs: análise de mídias para uma educação em rede. Em Rede - Revista de Educação à Distância, 2(1), $140-154$.

Xavier, F. et al. (2020) Análise de redes sociais como estratégia de apoio à vigilância em saúde durante a Covid-19. Estudos Avançados, 44(99), 261-282. 